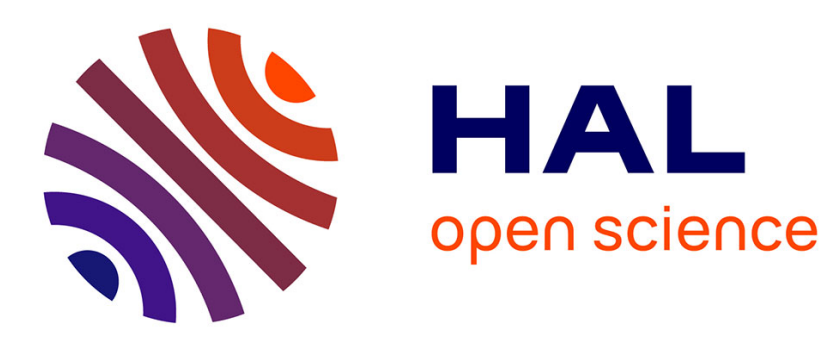

\title{
A cut-cell Hybrid High-Order method for elliptic problems with curved boundaries
}

Erik Burman, Alexandre Ern

\section{To cite this version:}

Erik Burman, Alexandre Ern. A cut-cell Hybrid High-Order method for elliptic problems with curved boundaries. ENUMATH 2017, Sep 2017, Bergen, Norway. 10.1007/978-3-319-96415-7_14. hal$01653685 \mathrm{v} 2$

\section{HAL Id: hal-01653685 \\ https://hal.science/hal-01653685v2}

Submitted on 6 Mar 2018

HAL is a multi-disciplinary open access archive for the deposit and dissemination of scientific research documents, whether they are published or not. The documents may come from teaching and research institutions in France or abroad, or from public or private research centers.
L'archive ouverte pluridisciplinaire HAL, est destinée au dépôt et à la diffusion de documents scientifiques de niveau recherche, publiés ou non, émanant des établissements d'enseignement et de recherche français ou étrangers, des laboratoires publics ou privés. 


\title{
A cut-cell Hybrid High-Order method for elliptic problems with curved boundaries
}

\author{
Erik Burman* $\quad$ Alexandre Ern $^{\dagger}$
}

\begin{abstract}
We design a Hybrid High-Order method for elliptic problems on curved domains. The method uses a cut cell technique for the representation of the curved boundary and imposes Dirichlet boundary conditions using Nitsche's method. The physical boundary can cut through the cells in a very general fashion and the method leads to optimal error estimates in the $H^{1}$-norm.
\end{abstract}

\section{Introduction}

The Hybrid High-Order (HHO) method has been recently introduced in [6, 7]. The idea is to approximate the solution using cell and face unknowns. The cell unknowns can be eliminated locally in each mesh cell, leading to a global problem coupling only the face unknowns. The HHO method is devised from a local reconstruction operator and a stabilization operator coupling the cell and face unknowns in each mesh cell. This leads to a discretization method that supports general meshes (with polyhedral cells and non-matching interfaces), is locally conservative, and delivers energy-norm error estimates of order $(k+1)$ (and $L^{2}$-norm error estimates of order $(k+2)$ under full elliptic regularity) using polynomials of order $k$ for the face unknowns and of order $l \in\{k-1, k, k+1\}$ for the cell unknowns.

The use of polyhedral meshes can simplify the meshing problem in many situations. Nevertheless, in some cases it can still be convenient to avoid building meshes fitted to the domain boundary. This is typically the case when the boundary changes during the computation, as in free-boundary problems or optimisation problems, or when the boundary is curved and high-order accuracy is to be retained. In classical finite element methods, fictitious domain approaches [8], where the computational mesh does not respect the domain boundary, are often efficient, but at the expense of accuracy. In order to improve the accuracy, unfitted finite element methods were introduced [1,9]. A well-known difficulty for unfitted finite element methods is that the conditioning of the linear system resulting from discretization has a strong dependence on the geometry of the intersection of the physical boundary with the mesh cells. Unfavorably cut cells, that is, cells having a small intersection with the physical domain,

\footnotetext{
*Department of Mathematics, University College London, London, UK-WC1E 6BT, UK, e.burman@ucl.ac.uk

${ }^{\dagger}$ Université Paris-Est, CERMICS (ENPC), 77455 Marne-la-Vallée cedex 2, and INRIA Paris, 75589 Paris, France, alexandre.ern@enpc.fr
} 
lead to ill-conditioning. In the case of $H^{1}$-conforming methods, this problem is typically solved by adding a penalty term that weakly couples the polynomial approximation in adjacent cells [3]. When using discontinuous approximation, another approach was proposed in [10] in the context of elliptic interface problems, where any unfavorably cut cell is merged with a neighboring cell having a favorable cut. This local agglomeration procedure generally leads to meshes having polyhedral cells.

In this work, we consider a (simple) elliptic PDE posed on an open, bounded, Lipschitz set $\Omega^{\prime}$ in $\mathbb{R}^{d}$ with a non-homogeneous Dirichlet condition on $\Gamma:=\partial \Omega^{\prime}$ :

$$
\begin{aligned}
-\nabla \cdot(\kappa \nabla u) & =f, & & \text { in } \Omega^{\prime}, \\
u & =g_{\mathrm{D}} & & \text { on } \Gamma .
\end{aligned}
$$

Here $f \in L^{2}\left(\Omega^{\prime}\right), g_{\mathrm{D}} \in H^{\frac{1}{2}}(\Gamma)$ and $\kappa$ is a positive, piecewise constant coefficient. We assume that $\Omega^{\prime}$ has a curved boundary $\Gamma$, and we want to avoid meshing $\Omega^{\prime}$ using cells with curved faces. One possible way to avoid this is to consider a fictitious domain approach. We embed $\Omega^{\prime}$ into a larger polyhedron $\Omega$ that can be meshed easily (by definition, a polyhedron is a finite union of simplices and has therefore a piecewise planar boundary). This mesh of $\Omega$ can be composed of cells having simple shapes (simplices, prisms, hexahedra), but can also contain more general polyhedral shapes. Our goal is to devise a cut-cell HHO method to approximate (1) using an unfitted mesh of the fictitious domain $\Omega$. For the imposition of the Dirichlet boundary condition, we use Nitsche's method, and robustness with respect to the cuts is ensured by using a local mesh agglomeration procedure reminiscent of that proposed in [10]. Owing to the local cell agglomeration, the resulting mesh of $\Omega$ is generally of polyhedral type. The present cut-cell HHO method and its analysis are adapted from the method devised in [4] for elliptic interface problems. An alternative HHO method for elliptic problems on curved meshes has been proposed recently in [2] using nonlinear mappings for the face unknowns and increasing the order of the reference face polynomials.

An outline of the paper is as follows. In the next section, we present the discrete framework, technical results, and the main assumptions. In Section 3, we introduce the cut-cell HHO method. Finally, we present an error estimate in Section 4.

\section{Discrete setting}

Let $\left(\mathcal{T}_{h}\right)_{h>0}$ be a shape-regular family of matching meshes covering $\Omega$ exactly, where the index $h$ refers to the maximal diameter of the mesh cells. For simplicity, we assume that the diffusion coefficient is piecewise constant on $\mathcal{T}_{h}$ and we let $\kappa_{T}$ denote its value on $T \in \mathcal{T}_{h}$. The meshes can have polyhedral cells, and the mesh cells are considered by convention to be open subsets of $\mathbb{R}^{d}$. The shape-regularity criterion for polyhedral meshes is that they admit a matching simplicial sub-mesh that satisfies the usual shape-regularity criterion in the sense of Ciarlet and such that each sub-cell (resp., sub-face) belongs to only one mesh cell (resp., at most one mesh face) having uniformly the same diameter. The shape-regularity of the mesh sequence is quantified by a parameter 
$\rho \in(0,1)$. In what follows, $B(\boldsymbol{y}, a)$ denotes the open ball with center $\boldsymbol{y}$ and radius $a$. For a subset $S \subset \mathbb{R}^{d}, h_{S}$ denotes the diameter of $S$.

\subsection{Notation for unfitted meshes}

For coherence of our notation, let us set $\Omega^{\backslash \Gamma}:=\Omega^{\prime}$. Let us define the partition $\mathcal{T}_{h}=\mathcal{T}_{h}^{\backslash \Gamma} \cup \mathcal{T}_{h}^{\Gamma} \cup \mathcal{T}_{h}^{c}$, where the subsets

$$
\begin{aligned}
\mathcal{T}_{h}^{\backslash \Gamma} & :=\left\{T \in \mathcal{T}_{h} \mid T \subset \Omega^{\backslash \Gamma}\right\}, \\
\mathcal{T}_{h}^{\Gamma} & :=\left\{T \in \mathcal{T}_{h} \mid \operatorname{meas}_{d-1}(T \cap \Gamma)>0\right\},
\end{aligned}
$$

collect, respectively, the mesh cells inside the physical domain $\Omega^{\backslash \Gamma}$ and the mesh cells cut by the physical boundary $\Gamma$. The mesh cells in $\mathcal{T}_{h}^{c}$ do not play any role in what follows so that we can discard them without loss of generality. Similarly, the mesh faces are collected in the set $\mathcal{F}_{h}$ which is partitioned into $\mathcal{F}_{h}=\mathcal{F}_{h}^{\backslash \Gamma} \cup \mathcal{F}_{h}^{\Gamma}$, where $\mathcal{F}_{h}^{\backslash \Gamma}$ collects the mesh faces inside the physical domain $\Omega^{\backslash \Gamma}$ and $\mathcal{F}_{h}^{\Gamma}$ collects the mesh faces cut by the physical boundary. For any mesh cell $T \in \mathcal{T}_{h}^{\Gamma}$, we define $T^{\backslash \Gamma}:=T \cap \Omega^{\backslash \Gamma}$ and $T^{\Gamma}:=T \cap \Gamma$. The boundary of $T^{\backslash \Gamma}$ is decomposed as $\partial T^{\backslash \Gamma}=(\partial T)^{\backslash \Gamma} \cup T^{\Gamma}$, where $(\partial T)^{\backslash \Gamma}:=\partial T \cap \Omega^{\backslash \Gamma}$ (see Figure 2 below).

\subsection{Admissible meshes}

The cut-cell HHO method is to be deployed on meshes satisfying two assumptions. Assumption 2.1 means that the physical boundary is well resolved by the mesh; this assumption is quantified by a regularity parameter $\gamma \in(0,1)$. Assumption 2.2 means that all the mesh cells in $\mathcal{T}_{h}^{\Gamma}$ are cut favorably by the physical boundary; this property is quantified by a cut parameter $\delta \in(0,1)$.

Assumption 2.1 (Resolving $\Gamma$ ). There is $\gamma \in(0,1)$ s.t. for all $T \in \mathcal{T}_{h}^{\Gamma}$, there is a point $\hat{\boldsymbol{x}}_{T} \in \mathbb{R}^{d}$ so that, for all $\boldsymbol{s} \in T^{\Gamma},\left\|\hat{\boldsymbol{x}}_{T}-\boldsymbol{s}\right\|_{\ell^{2}} \leq \gamma^{-1} h_{T}$ and $d\left(\hat{\boldsymbol{x}}_{T}, T_{\boldsymbol{s}} \Gamma\right) \geq$ $\gamma h_{T}$ where $T_{\boldsymbol{s}} \Gamma$ is the tangent plane to $\Gamma$ at the point $\boldsymbol{s}$.

Assumption 2.2 (Cut cells). There is $\delta \in(0,1)$ such that, for all $T \in \mathcal{T}_{h}^{\Gamma}$, there is $\tilde{\boldsymbol{x}}_{T} \in T^{\backslash \Gamma}$ so that $B\left(\tilde{\boldsymbol{x}}_{T}, \delta h_{T}\right) \subset T^{\backslash \Gamma}$.

Let $\tilde{\mathcal{T}}_{h}$ be an initially given mesh from a shape-regular sequence of (polyhedral) meshes. It is shown in [4, Lemma 6.1] that the Assumption 2.1 is satisfied provided $\tilde{\mathcal{T}}_{h}$ is fine enough. To ensure that the Assumption 2.2 is also satisfied, we identify those mesh cells in $\tilde{\mathcal{T}}_{h}^{\Gamma}$ (with obvious notation) for which the assumption fails and agglomerate them with neighboring elements satisfying the assumption. The agglomeration procedure is as follows, where for all $T \in \tilde{\mathcal{T}}_{h}$, we define the set of elements with non-empty intersection with $T$ as $\Delta(T):=\left\{T^{\prime} \in \tilde{\mathcal{T}}_{h} \mid T \cap T^{\prime} \neq \emptyset\right\}:$

1. Partition $\tilde{\mathcal{T}}_{h}^{\Gamma}$ into $\tilde{\mathcal{T}}_{h}^{\mathrm{KO}} \cup \tilde{\mathcal{T}}_{h}^{\mathrm{OK}}$ so that $\tilde{T} \in \tilde{\mathcal{T}}_{h}^{\mathrm{OK}}$ iff there is $\boldsymbol{x}_{\tilde{T}} \in \tilde{T}$ s.t. the ball $B\left(\boldsymbol{x}_{\tilde{T}}, \delta_{0} h_{\tilde{T}}\right)$ is in $\tilde{T}^{\backslash \Gamma}=\tilde{T} \cap \Omega^{\backslash \Gamma}$, whereas $\tilde{T} \in \tilde{\mathcal{T}}_{h}^{\text {ko }}$ otherwise.

2. For all $\tilde{T} \in \tilde{\mathcal{T}}_{h}^{\text {Ko }}$, we choose a neighboring mesh cell $N(\tilde{T})$ so that

$$
N(\tilde{T}) \in\left(\tilde{\mathcal{T}}_{h}^{\mathrm{OK}} \cup \tilde{\mathcal{T}}_{h}^{\backslash \Gamma}\right) \cap \Delta(\tilde{T}) .
$$


It is shown in [4] that this set is nonempty if the mesh $\tilde{\mathcal{T}}_{h}$ is fine enough and if the initial cut parameter $\delta_{0}$ is small enough.

3. Let $N\left(\tilde{\mathcal{T}}_{h}^{\text {KO }}\right)$ be the collection of all the cells in $\tilde{\mathcal{T}}_{h}^{\text {OK }} \cup \tilde{\mathcal{T}}_{h}^{\backslash \Gamma}$ that have been selected at least once in Step 2. For all $\tilde{T}^{\sharp} \in N\left(\tilde{\mathcal{T}}_{h}^{\text {KO }}\right)$, we define the agglomerated cell $\tilde{T}^{\sharp} \cup\left\{\tilde{T} \in \tilde{\mathcal{T}}_{h}^{\mathrm{KO}} \mid N(\tilde{T})=\tilde{T}^{\sharp}\right\}$.

4. We collect the agglomerated cells in $\tilde{\mathcal{T}}_{h}^{\text {agglo }}$ and define the new mesh as

$$
\mathcal{T}_{h}:=\left(\left(\tilde{\mathcal{T}}_{h}^{\mathrm{OK}} \cup \tilde{\mathcal{T}}_{h}^{\backslash \Gamma}\right) \backslash N\left(\tilde{\mathcal{T}}_{h}^{\mathrm{KO}}\right)\right) \cup \tilde{\mathcal{T}}_{h}^{\text {agglo }} .
$$

It is shown in [4] that the new mesh $\mathcal{T}_{h}$ is still shape-regular (with possibly a smaller value of the parameter $\rho$ ), still satisfies the Assumption 2.1 (with possibly a smaller parameter $\gamma$ ), and additionally satisfies the Assumption 2.2 (with possibly a parameter $\delta$ smaller than $\delta_{0}$ ).

\subsection{Trace inequalities}

The following two trace inequalities, which are crucial for the robustness of the error estimate (20) (see below), hinge on Assumptions 2.1 and 2.2. For a proof, we refer the reader to [4, Lemma 3.3 and 3.4].

Lemma 2.1 (Multiplicative trace inequality). There are $c_{\mathrm{mtr}}>0$ and $\theta_{\mathrm{mtr}} \geq 1$, depending on $\rho$ and $\gamma$, such that, for all $T \in \mathcal{T}_{h}^{\Gamma}$, there is $\check{\boldsymbol{x}}_{T} \in T$ so that, for all $v \in H^{1}\left(T^{\dagger}\right)$ with $T^{\dagger}=B\left(\check{\boldsymbol{x}}_{T}, \theta_{\mathrm{mtr}} h_{T}\right)$,

$$
\|v\|_{L^{2}(\partial T \backslash \Gamma)} \leq c_{\mathrm{mtr}}\left(h_{T}^{-\frac{1}{2}}\|v\|_{L^{2}\left(T^{\dagger}\right)}+\|v\|_{L^{2}\left(T^{\dagger}\right)}^{\frac{1}{2}}\|\nabla v\|_{L^{2}\left(T^{\dagger}\right)}^{\frac{1}{2}}\right) .
$$

Lemma 2.2 (Discrete trace inequality). Let $l \in \mathbb{N}, l \geq 0$. There is $c_{\mathrm{dtr}}$, depending on $l, \rho$, and $\delta$, such that, for all $T \in \mathcal{T}_{h}^{\Gamma}$, and all $v \in \mathbb{P}_{d}^{l}\left(T^{\backslash \Gamma}\right)$,

$$
\|v\|_{L^{2}(\partial T \backslash \Gamma)} \leq c_{\mathrm{dtr}} h_{T}^{-\frac{1}{2}}\|v\|_{L^{2}(T \backslash \Gamma)} .
$$

\section{The cut-cell HHO method}

In this section, we describe the cut-cell HHO method for the ficitious domain problem. Let $k \geq 0$ be the polynomial degree for the face unknowns; the polynomial degree for the cell unknowns is $(k+1)$. For any mesh cell $T \in \mathcal{T}_{h}$, the set $\mathcal{F}_{\partial T}$ is the collection of the faces of $T$. Whenever $T \in \mathcal{T}_{h}^{\Gamma}$, the set $\mathcal{F}_{\partial T}^{\backslash \Gamma}=\left\{F^{\backslash \Gamma}=F \cap \Omega^{\backslash \Gamma} \mid F \in \mathcal{F}_{T}\right.$, meas $\left._{d-1}\left(F^{\backslash \Gamma}\right)>0\right\}$ is the collection of the faces and cut faces of $T$ that partition the boundary $(\partial T)^{\backslash \Gamma}$.

\subsection{Uncut cells}

Let $T \in \mathcal{T}_{h}^{\backslash \Gamma}$. The local unknowns form a pair composed of one polynomial of order $(k+1)$ in $T$ and a piecewise polynomial of order $k$ on $\partial T$. The local unknowns are generically denoted as

$$
\hat{v}_{T}=\left(v_{T}, v_{\partial T}\right) \in \mathbb{P}_{d}^{k+1}(T) \times \mathbb{P}_{d-1}^{k}\left(\mathcal{F}_{\partial T}\right)=: \hat{\mathcal{X}}_{T},
$$



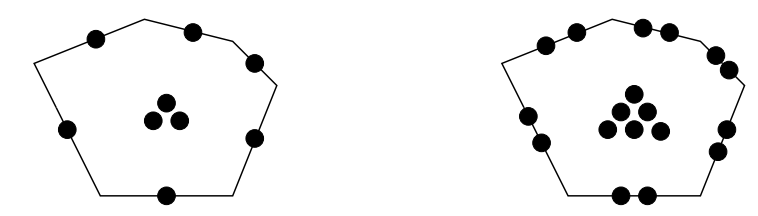

Figure 1: Uncut hexagonal cell. Left: $k=0$; Right: $k=1$. Each dot symbolizes one degree of freedom (not necessarily a pointwise evaluation).

with $\mathbb{P}_{d-1}^{k}\left(\mathcal{F}_{\partial T}\right)=X_{F \in \mathcal{F}_{\partial T}} \mathbb{P}_{d-1}^{k}(F)$. The placement of the discrete unknowns for the uncut cells is illustrated in Figure 1.

We define the following local bilinear form for all $v, w \in H^{1}(T)$ :

$$
a_{T}(v, w)=\int_{T} \kappa_{T} \nabla v \cdot \nabla w
$$

There are two ingredients to devise the local HHO bilinear form. The first one is a reconstruction operator. Let $\hat{v}_{T}=\left(v_{T}, v_{\partial T}\right) \in \hat{\mathcal{X}}_{T}$. Then, we reconstruct a polynomial $r_{T}^{k+1}\left(\hat{v}_{T}\right) \in \mathbb{P}_{d}^{k+1}(T)$ by requiring that, for all $z \in \mathbb{P}_{d}^{k+1}(T)$, the following holds true:

$$
a_{T}\left(r_{T}^{k+1}\left(\hat{v}_{T}\right), z\right)=a_{T}\left(v_{T}, z\right)-\int_{\partial T} \kappa_{T} \nabla z \cdot \boldsymbol{n}_{T}\left(v_{T}-v_{\partial T}\right),
$$

where $\boldsymbol{n}_{T}$ is the unit outward-pointing normal to $T$. Note that $r_{T}^{k+1}\left(\hat{v}_{T}\right)$ is uniquely defined up to an additive constant; one way to fix the constant is to prescribe $\int_{T} r_{T}^{k+1}\left(\hat{v}_{T}\right)=\int_{T} v_{T}$. The second ingredient is the stabilization bilinear form defined so that, for all $\hat{v}_{T}, \hat{w}_{T} \in \hat{\mathcal{X}}_{T}$,

$$
s_{T}\left(\hat{v}_{T}, \hat{w}_{T}\right)=\kappa_{T} h_{T}^{-1} \int_{\partial T} \Pi_{\partial T}^{k}\left(v_{T}-v_{\partial T}\right)\left(w_{T}-w_{\partial T}\right),
$$

where $\Pi_{\partial T}^{k}$ denotes the $L^{2}$-orthogonal projector onto the piecewise polynomial space $\mathbb{P}_{d-1}^{k}\left(\mathcal{F}_{\partial T}\right)$. Finally, the local HHO bilinear and linear forms to be used when assembling the global discrete problem are as follows: For all $\hat{v}_{T}, \hat{w}_{T} \in \hat{\mathcal{X}}_{T}$,

$$
\begin{aligned}
\hat{a}_{T}^{\backslash \Gamma}\left(\hat{v}_{T}, \hat{w}_{T}\right) & =a_{T}\left(r_{T}^{k+1}\left(\hat{v}_{T}\right), r_{T}^{k+1}\left(\hat{w}_{T}\right)\right)+s_{T}\left(\hat{v}_{T}, \hat{w}_{T}\right), \\
\hat{\ell}_{T}^{\backslash \Gamma}\left(\hat{w}_{T}\right) & =\int_{T} f w_{T} .
\end{aligned}
$$

\subsection{Cut cells: fictitious domain problem}

Let $T \in \mathcal{T}_{h}^{\Gamma}$. We use capital letters to denote a generic function $V \in H^{1}\left(T^{\backslash \Gamma}\right)$. The unit outward-pointing normal to $\Gamma$ is denoted $\boldsymbol{n}$. We define the following Nitsche bilinear form for all $V, W \in H^{s}\left(T^{\backslash \Gamma}\right), s>\frac{3}{2}$ :

$n_{T}(V, W)=\int_{T \backslash \Gamma} \kappa_{T} \nabla V \cdot \nabla W-\int_{T^{\Gamma}}\left\{\left(\kappa_{T} \nabla V \cdot \boldsymbol{n}\right) W+\left(\kappa_{T} \nabla W \cdot \boldsymbol{n}\right) V-\eta \frac{\kappa_{T}}{h_{T}} V W\right\}$, 

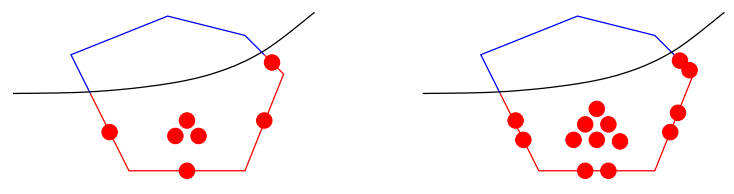

Figure 2: Cut hexagonal cell. The physical domain $\Omega^{\backslash \Gamma}$ (red/light grey) is located below the curved boundary $\Gamma$ so that $(\partial T)^{\backslash \Gamma}$ is composed of two faces and two cut faces. Left: $k=0$; Right: $k=1$. Each dot symbolizes one degree of freedom (not necessarily a pointwise evaluation).

where the user-specified parameter $\eta$ is such that $\eta \geq 4 c_{\mathrm{dtr}}^{2}$ where $c_{\mathrm{dtr}}$ results from the discrete trace inequality (6) with polynomial degree $l=k$ (this follows using standard arguments for the stability of Nitsche's method, see [4, Lemma 5.1]). The local HHO unknowns are

$$
\hat{V}_{T}=\left(V_{T}, V_{\partial T}\right) \in \hat{\mathcal{X}}_{T}:=\mathbb{P}_{d}^{k+1}\left(T^{\backslash \Gamma}\right) \times \mathbb{P}_{d-1}^{k}\left(\mathcal{F}_{\partial T}^{\backslash \Gamma}\right),
$$

where $\mathbb{P}_{d-1}^{k}\left(\mathcal{F}_{\partial T}^{\backslash \Gamma}\right)$ is the piecewise polynomial space of order $k$ on $(\partial T)^{\backslash \Gamma}$ based on the (sub-)faces in $\mathcal{F}_{\partial T}^{\backslash \Gamma}$; note that we do not introduce discrete unknowns on $T^{\Gamma}$. The placement of the discrete HHO unknowns in the cut cells is illustrated in Figure 2.

As for the uncut cells, there are two key ingredients to devise the local HHO bilinear form: reconstruction and stabilization. Let $\hat{V}_{T} \in \hat{\mathcal{X}}_{T}$. Then, we reconstruct a polynomial $R_{T}^{k+1}\left(\hat{V}_{T}\right) \in \mathbb{P}_{d}^{k+1}\left(T^{\backslash \Gamma}\right)$ by requiring that, for all $Z \in \mathbb{P}_{d}^{k+1}\left(T^{\backslash \Gamma}\right)$, the following holds true:

$$
n_{T}\left(R_{T}^{k+1}\left(\hat{V}_{T}\right), Z\right)=n_{T}\left(V_{T}, Z\right)-\int_{(\partial T) \backslash \Gamma} \kappa_{T} \nabla Z \cdot \boldsymbol{n}_{T}\left(V_{T}-V_{\partial T}\right) .
$$

It follows from the stability of Nitsche's method that $R_{T}^{k+1}\left(\hat{V}_{T}\right)$ is uniquely defined by (14) up to an additive constant; one way to fix the constant is to prescribe $\int_{T \backslash \Gamma} R_{T}^{k+1}\left(\hat{V}_{T}\right)=\int_{T \backslash \Gamma} V_{T}$. Concerning stabilization, we set for all $\hat{V}_{T}, \hat{W}_{T} \in \hat{\mathcal{X}}_{T}$,

$$
s_{T}\left(\hat{V}_{T}, \hat{W}_{T}\right)=\kappa_{T} h_{T}^{-1} \int_{(\partial T) \backslash \Gamma} \Pi_{(\partial T) \backslash \Gamma}^{k}\left(V_{T}-V_{\partial T}\right)\left(W_{T}-W_{\partial T}\right),
$$

where $\Pi_{(\partial T) \backslash \Gamma}^{k}$ denotes the $L^{2}$-orthogonal projector onto the piecewise polynomial space $\mathbb{P}_{d-1}^{k}\left(\mathcal{F}_{\partial T}^{\backslash \Gamma}\right)$. Finally, the local HHO bilinear and linear forms are as follows: For all $\hat{V}_{T}, \hat{W}_{T} \in \hat{\mathcal{X}}_{T}$,

$$
\begin{aligned}
\hat{a}_{T}^{\Gamma}\left(\hat{V}_{T}, \hat{W}_{T}\right) & =n_{T}\left(R_{T}^{k+1}\left(\hat{V}_{T}\right), R_{T}^{k+1}\left(\hat{W}_{T}\right)\right)+s_{T}\left(\hat{V}_{T}, \hat{W}_{T}\right), \\
\hat{\ell}_{T}^{\Gamma}\left(\hat{W}_{T}\right) & =\int_{T \backslash \Gamma} f W_{T}+\int_{T^{\Gamma}} g_{\mathrm{D}} \phi_{T}\left(W_{T}\right),
\end{aligned}
$$

with $\phi_{T}\left(W_{T}\right)=-\kappa_{T} \nabla W_{T} \cdot \boldsymbol{n}_{\Gamma}+\eta \kappa_{T} h_{T}^{-1} W_{T}$. 


\subsection{The global formulation}

To formulate the global discrete problem, we introduce the sets $\hat{\mathcal{T}}_{h}:=\mathcal{T}_{h}^{\backslash \Gamma} \cup$ $\left\{T \cap \Omega^{\backslash \Gamma} \mid T \in \mathcal{T}_{h}^{\Gamma}\right\}$ and $\hat{\mathcal{F}}_{h}:=\mathcal{F}_{h}^{\backslash \Gamma} \cup\left\{F \cap \Omega^{\backslash \Gamma} \mid F \in \mathcal{F}_{h}^{\Gamma}\right\}$, and we set

$$
\hat{\mathcal{X}}_{h}:=X_{T \in \hat{\mathcal{T}}_{h}} \mathbb{P}_{d}^{k+1}(T) \times \mathrm{X}_{F \in \hat{\mathcal{F}}_{h}} \mathbb{P}_{d-1}^{k}(F) .
$$

Let $\hat{V}_{h} \in \hat{\mathcal{X}}_{h}$. For all $T \in \mathcal{T}_{h}^{\backslash \Gamma}$, we denote $\hat{v}_{T}=\left(v_{T}, v_{\partial T}\right) \in \hat{\mathcal{X}}_{T}$ the components of $\hat{V}_{h}$ attached to the uncut cell $T$. For all $T \in \mathcal{T}_{h}^{\Gamma}$, we denote $\hat{V}_{T}=\left(V_{T}, V_{\partial T}\right) \in$ $\hat{\mathcal{X}}_{T}$ the components of $\hat{V}_{h}$ attached to the cut cell $T$. The global discrete problem then reads as follows: Find $\hat{U}_{h} \in \hat{\mathcal{X}}_{h}$ s.t.

$$
\hat{a}_{h}\left(\hat{U}_{h}, \hat{W}_{h}\right)=\hat{\ell}_{h}\left(\hat{W}_{h}\right), \quad \forall \hat{W}_{h} \in \hat{\mathcal{X}}_{h}
$$

with

$$
\begin{aligned}
\hat{a}_{h}\left(\hat{V}_{h}, \hat{W}_{h}\right) & =\sum_{T \in \mathcal{T}_{h}^{\backslash \Gamma}} \hat{a}_{T}^{\backslash \Gamma}\left(\hat{v}_{T}, \hat{w}_{T}\right)+\sum_{T \in \mathcal{T}_{h}^{\Gamma}} \hat{a}_{T}^{\Gamma}\left(\hat{V}_{T}, \hat{W}_{T}\right), \\
\hat{\ell}_{h}\left(\hat{W}_{h}\right) & =\sum_{T \in \mathcal{T}_{h}^{\backslash \Gamma}} \hat{\ell}_{T}^{\backslash \Gamma}\left(\hat{w}_{T}\right)+\sum_{T \in \mathcal{T}_{h}^{\Gamma}} \hat{\ell}_{T}^{\Gamma}\left(\hat{W}_{T}\right),
\end{aligned}
$$

where $\hat{a}_{T}^{\backslash \Gamma}(\cdot, \cdot)$ and $\hat{\ell}_{T}^{\backslash \Gamma}(\cdot)$ are defined by (11) for all $T \in \mathcal{T}_{h}^{\backslash \Gamma}$ and $\hat{a}_{T}^{\Gamma}(\cdot, \cdot)$ and $\hat{\ell}_{T}^{\Gamma}(\cdot)$ are defined by $(16)$ for all $T \in \mathcal{T}_{h}^{\Gamma}$.

\section{Main result: error estimate}

We now state our main result on the error analysis. The proof follows by adapting the arguments of $[4$, Thm. 5.9].

Theorem 4.1 (Error estimate). Assume that $u \in H^{k+2}\left(\Omega^{\backslash \Gamma}\right), k \geq 0$, is the solution to (1). Let $\hat{U}_{h} \in \hat{\mathcal{X}}_{h}$ solve (18). Then, the following bound holds true:

$$
\begin{aligned}
\sum_{T \in \mathcal{T}_{h}^{\backslash \Gamma}}\left\|\nabla\left(u-u_{T}\right)\right\|_{T}^{2} & +\sum_{T \in \mathcal{T}_{h}^{\Gamma}}\left\|\nabla\left(u-U_{T}\right)\right\|_{T \backslash \Gamma}^{2} \\
& +\sum_{T \in \mathcal{T}_{h}^{\Gamma}} h_{T}^{-1}\left\|g_{\mathrm{D}}-U_{T}\right\|_{T^{\Gamma}}^{2} \lesssim h^{2(k+1)}|u|_{H^{k+2}\left(\Omega^{\backslash \Gamma}\right)}^{2} .
\end{aligned}
$$

\section{Acknowledgments}

The first author was partly supported by EPSRC Grant EP/P01576X/1. This work was initiated when the authors were visiting the Institut Henri Poincaré during the Fall 2016 Thematic Trimester "Numerical Methods for Partial Differential Equations". The support of IHP is gratefully acknowledged.

\section{References}

[1] J. W. BARRett AND C. M. Elliott, Fitted and unfitted finite-element methods for elliptic equations with smooth interfaces, IMA J. Numer. Anal. 7:3 (1987), 283-300. 
[2] L. Botti And D. A. Di Pietro, Assessment of Hybrid High-Order methods on curved meshes and comparison with discontinuous Galerkin methods, HAL e-print hal-01581883 (2017).

[3] E. Burman, Ghost penalty, C. R. Math. Acad. Sci. Paris 348:21-22 (2010), $1217-1220$

[4] E. Burman And A. ERn, An unfitted Hybrid High-Order method for elliptic interface problems, ArXiv e-print 1710.10132 (2017).

[5] B. Cockburn, J. Gopalakrishnan, and R. Lazarov, Unified hybridization of discontinuous Galerkin, mixed, and continuous Galerkin methods for second order elliptic problems, SIAM J. Numer. Anal. 47:2 (2009), 1319-1365.

[6] D. A. Di Pietro, A. Enn, A Hybrid High-Order locking-free method for linear elasticity on general meshes, Comput. Meth. Appl. Mech. Engrg., 283:1, 2015 .

[7] D. A. Di Pietro, A. Ern, And S. Lemaire, An arbitrary-order and compact-stencil discretization of diffusion on general meshes based on local reconstruction operators, Comput. Meth. Appl. Math. 14:4 (2014), 461472 .

[8] V. Girault and R. Glowinski, Error analysis of a fictitious domain method applied to a Dirichlet problem, Japan J. Indust. Appl. Math. 12:3 (1995), 487-514.

[9] A. Hansbo and P. Hansbo, An unfitted finite element method, based on Nitsche's method, for elliptic interface problems, Comput. Methods Appl. Mech. Engrg. 191:47-48 (2002), 5537-5552.

[10] A. Johansson and M. G. LaRson, A high order discontinuous Galerkin Nitsche method for elliptic problems with fictitious boundary, Numer. Math. 123:4 (2013), 607-628. 